\title{
Discrete-Time Expectation Maximization Algorithms for Markov-Modulated Poisson Processes
}

\author{
Robert J. Elliott and W. P. Malcolm
}

Abstract-In this paper, we consider parameter estimation Markov-modulated Poisson processes via robust filtering and smoothing techniques. Using the expectation maximization algorithm framework, our filters and smoothers can be applied to estimate the parameters of our model in either an online configuration or an offline configuration. Further, our estimator dynamics do not involve stochastic integrals and our new formulas, in terms of time integrals, are easily discretized, and are written in numerically stable forms in W. P. Malcolm, R. J. Elliott, and J. van der Hoek, "On the numerical stability of time-discretized state estimation via clark transformations," presented at the IEEE Conf. Decision Control, Mauii, HI, Dec. 2003.

Index Terms-Change of measure, counting processes, expectation maximization (EM) algorithm, martingales.

$\mathbf{T}$ HE WELL-KNOWN expectation maximization (EM) algorithm [8], [17] provides a scheme for solving a problem common in signal processing: estimating the parameters of a probability distribution for a known, partially observed dynamical system. This problem has received considerable attention for common signal models, such as the discrete-time Gaus-Markov model or the observation of a Markov process through a Brownian motion, [10], [24]. In this paper, we propose EM algorithms for the so-called Markov-modulated Poisson process (MMPP).

A MMPP is conditionally a Poisson counting process, whose rate of arrivals depends upon the state of an indirectly observed Markov chain. These models have enjoyed many successful applications in queueing theory, and more recently, have been studied in the context of packet traffic estimation, and biomedical and optical-signal processing.

Since our hidden-state process models are continuous-time Markov chains, the parameter estimation problem we consider, concerns computing estimates for the rate matrix of the Markov chain and the vector of Poisson intensities for the observation process. Traditionally, the EM algorithm is implemented by maximizing a log-likelihood function over a parameter space [11], [21], [22]. In some applications, this approach can lead to

Manuscript received Jun 7, 2004; revised December 9, 2005 and December 1, 2006. Recommended by Associate Editor xxxx. The work of W. P. Malcolm was supported by the National Information and Communication Technology (ICT) Australia under the Grant given by the Australian Government's Department of Communications, Information Technology and the Arts and the Australian Research Council through Backing Australia's Ability and the ICT Centre of Excellence program.

R. J. Elliott was with the Haskayne School of Business, University of Calgary, Calgary, AB T2N 1N4, Canada. He is now with the University of Alberta, Edmonton, AB T6G 2E1, Canada (e-mail: relliott@ucalgary.ca).

W. P. Malcolm is with the Statistical Machine Learning Program of the National Information and Communication Technology (ICT) Australia (NICTA), Canberra, A.C.T. 2601, Australia (e-mail: paul.malcolm@nicta.com.au).

Digital Object Identifier 10.1109/TAC.2007.914305 technical difficulties. For example, the form of the log-likelihood function could be complicated or the operation of maximization of this function might be difficult.

The implementations of the EM algorithms we present are the so-called filter-based and smoother-based EM algorithm [4], [10]. In the filter-based scheme, the parameter estimates are computed online by running a set of four recursive filters whose only storage requirements are previous estimates. Adapting the transformation techniques introduced by Clark [1], we compute the so-called robust versions of these filter, where the observation processes appear as parameters rather than as stochastic integrators. These formulations have been shown to have some numerical advantages [16]. Our smoother-based EM algorithm exploits a type of identity between the forward robust filter and its reverse-time counterpart. Smoothed estimates are obtained without recourse to stochastic integration.

The paper is organized as follows. In Section II, the signal models for the state and observation processes are defined; our reference probability measure is also defined in this section. In Section III, we briefly recall the EM algorithm and compute a filter-based EM algorithm for MMPPs. In this section, we also compute robust filter dynamics that do not include stochastic integrals. In Section IV, we compute a robust smoother-based EM algorithm for an MMPP. Finally, in Section V, we compute a discrete-time data-recursive smoother-based EM algorithm for an MMPP.

\section{DYNAMICS AND REFERENCE PROBABILITY}

Initially, we suppose that all processes are defined on the measurable space $(\Omega, \mathcal{F})$ with probability measure $P$.

\section{A. State Process Dynamics}

Suppose that the state process $X=\left\{X_{t}, 0 \leq t\right\}$ is a continuous-time finite-state Markov chain with rate matrix $A$ and an initial probability distribution $p_{0}$. We now use the well-known canonical representation for a Markov chain, that is, without loss of generality, the state space of $X$ is $\mathcal{L}=\left\{\boldsymbol{e}_{1}, \boldsymbol{e}_{2}, \ldots, \boldsymbol{e}_{n}\right\}$, where $\boldsymbol{e}_{i}$ denotes a column vector in $\mathbb{R}^{n}$ with unity in the $i$ th position and zero elsewhere. The dynamics for this process are

$$
X_{t}=X_{0}+\int_{0}^{t} A X_{u} d u+M_{t}
$$

Here, $M$ is a $\left(\sigma\left\{X_{t}, 0 \leq t\right\}, P\right)$-martingale and the matrix $A \in$ $\mathbb{R}^{n \times n}$ is a rate matrix for the process $X$. 


\section{B. Observation Process Dynamics}

Suppose that the state process $X$ is observed through a counting process whose Doob-Meyer decomposition is

$$
N_{t}=\int_{0}^{t}\left\langle X_{u}, \lambda\right\rangle d u+V_{t}
$$

Here, $V$ is a $\left(\sigma\left\{N_{u}, 0 \leq u \leq t\right\}, P\right)$-martingale, $\langle\cdot, \cdot\rangle$ denotes an inner product, and $\lambda \in \mathbb{R}_{+}^{n}$ is a vector of $n$ nonnegative Poisson intensities. Our filtrations are given by

$$
\begin{aligned}
\mathbb{F}_{t} & =\left\{\mathcal{F}_{t}\right\}, \quad \text { where } \mathcal{F}_{t} \triangleq \sigma\left\{X_{u} ; 0 \leq u \leq t\right\} \\
\mathbb{Y}_{0, t} & =\left\{\mathcal{Y}_{0, t}\right\}, \quad \text { where } \mathcal{Y}_{0, t} \triangleq \sigma\left\{N_{u} ; 0 \leq u \leq t\right\} \\
\mathbb{G}_{0, t} & =\left\{\mathcal{G}_{0, t}\right\}, \quad \text { where } \mathcal{G}_{0, t} \triangleq \sigma\left\{N_{u}, X_{u} ; 0 \leq u \leq t\right\} .
\end{aligned}
$$

\section{Reference Probability}

We define a probability measure $P^{\dagger}$ on the space $(\Omega, \mathcal{F})$ such that, under $P^{\dagger}$, the following two conditions hold.

1) The state process $X$ is a Markov process with intensity matrix $A$ and initial probability distribution $p_{0}$.

2) The observation process $N$ is a standard Poisson process, that is, $N$ has a fixed intensity of unity.

The real-world probability measure $P$ is defined by setting

$$
\left.\frac{d P}{d P^{\dagger}}\right|_{\mathcal{G}_{0, t}}=\Lambda_{0, t}
$$

where

$$
\begin{aligned}
\Lambda_{0, t} & =\prod_{0<u \leq t}\left\langle X_{u}, \lambda\right\rangle^{\Delta N_{u}} \exp \left\{\int_{0}^{t}\left(1-\left\langle X_{u}, \lambda\right\rangle\right) d u\right\} \\
& =1+\int_{0}^{t} \Lambda_{u-}\left(\left\langle X_{u}, \lambda\right\rangle-1\right)\left(d N_{u}-d u\right)
\end{aligned}
$$

Here,

$$
\begin{aligned}
\Delta N_{\tau} & \triangleq N_{\tau}-\lim _{\epsilon \downarrow 0} N_{\tau-\epsilon} \\
& =N_{\tau}-N_{\tau-} .
\end{aligned}
$$

Lemma 1: Under the measure $P$, the dynamics for the Markov process $X$ are unchanged and given by (2.1).

A proof of Lemma 1 is given in the Appendix. Further detail on the theory of Girsanov's theorem and its application to estimation problems for stochastic dynamical systems can be found in the texts [2] and [3].

\section{FILTER-BASED EM AlgORITHM}

\section{A. EM Algorithm}

The EM algorithm is a two-step iterative process for computing maximum likelihood (ML) estimates. This process is usually terminated when some imposed measure of convergence for the sequence of maximum likelihood estimators (MLEs) is satisfied. Let $\theta$ index a given family of probability measures $P_{\theta}$, where $\theta \in \Theta$. All such measures $P_{\theta}$ defined on a measurable space $(\Omega, \mathcal{F})$ are assumed absolutely continuous with respect to 111 a fixed probability measure $P$. Suppose $\mathcal{Y} \subset \mathcal{F}$.

The two iterative steps in the EM algorithm are as follows. 113

1) Expectation step: Fix $\theta^{*}=\widehat{\theta}_{\tau}$, then compute $Q\left(\cdot, \theta^{*}\right), \quad 114$ where

$$
Q\left(\theta, \theta^{*}\right)=E_{\theta^{*}}\left[\log \frac{d P_{\theta}}{d P_{\theta^{*}}} \mid \mathcal{Y}\right] .
$$

2) Maximization step: Maximize $Q\left(\theta, \theta^{*}\right)$ over the space $\Theta$

$$
\widehat{\theta}_{\tau+1} \in \underset{\theta \in \Theta}{\operatorname{argmax}} Q\left(\theta, \theta^{*}\right) \text {. }
$$

\section{B. State Estimation Filters}

The so-called filter-based form of the EM algorithm for a continuous-time Markov chain observed in Brownian motion was presented in [4] and a robust version is given in [10]. In this paper, we develop a version of the techniques used in [10] for parameter estimation with MMPPs. This method is based essentially on four quantities, each concerning the indirectly observed Markov process $X$ and each computed by using the information up to and including time $t$. We now list the four quantities of interest for the filter-based EM algorithm.

1) $X_{t}$, the state of the Markov chain. We are interested in $E\left[X_{t} \mid \mathcal{Y}_{t}\right]$. By Bayes' Theorem this is $E^{\dagger}\left[\Lambda_{t} X_{t} \mid \mathcal{Y}_{0, t}\right] / E^{\dagger}\left[\Lambda_{t} \mid \mathcal{Y}_{0, t}\right]$. Write

$$
q_{t} \triangleq E^{\dagger}\left[\Lambda_{t} X_{t} \mid \mathcal{Y}_{0, t}\right] \text {. }
$$

Then,

$$
\begin{aligned}
q_{t}= & q_{0}+\int_{0}^{t} A q_{u} d u \\
& +\int_{0}^{t} \operatorname{diag}\left\{\left\langle\lambda, \boldsymbol{e}_{\ell}\right\rangle-1\right\}\left(d N_{u}-d u\right) \in \mathbb{R}^{n} .
\end{aligned}
$$

Here,

$\operatorname{diag}\left\{\left\langle\lambda, e_{\ell}\right\rangle-1\right\}$

$$
=\left[\begin{array}{lll}
\left\langle\lambda, \boldsymbol{e}_{1}\right\rangle-1 & & \\
& \left\langle\lambda, \boldsymbol{e}_{2}\right\rangle-1 & \\
& \ddots & \\
& & \left\langle\lambda, \boldsymbol{e}_{n}\right\rangle-1
\end{array}\right]
$$

and $A$ is the rate matrix for the process $X$. The unnor- 132 malized probability $q$ is converted to its corresponding 133 normalized probability by noting that $\sum_{i=1}^{n}\left\langle X_{t}, \boldsymbol{e}_{i}\right\rangle=1, \quad 134$ so $E^{\dagger}\left[\Lambda_{t} \mid \mathcal{Y}_{t}\right]=\left\langle q_{t}, \mathbf{1}\right\rangle$. Here, $\mathbf{1}=(1,1, \ldots, 1)^{\prime} \in \mathbb{R}^{n}$. 135 Therefore,

$$
P\left(X_{t}=\boldsymbol{e}_{i} \mid \mathcal{Y}_{0, t}\right)=\frac{\left\langle q_{t}, \boldsymbol{e}_{i}\right\rangle}{\sum_{\ell=1}^{n}\left\langle q_{t}, \boldsymbol{e}_{\ell}\right\rangle} .
$$

A proof of (3.12) is given in the Appendix.

2) $J_{t}^{i}$, the cumulative sojourn time spent by the process $X$ in 138 state $e_{i}$ is

$$
J_{t}^{i}=\int_{0}^{t}\left\langle X_{u}, \boldsymbol{e}_{i}\right\rangle d u
$$


3) $N_{t}^{(j, i)}$, the number of transitions $\boldsymbol{e}_{i} \rightarrow \boldsymbol{e}_{j}$ of $X$, where $i \neq j$, up to time $t$ is

$$
N_{t}^{(j, i)}=\int_{0}^{t}\left\langle X_{u-}, \boldsymbol{e}_{i}\right\rangle\left\langle d X_{u}, \boldsymbol{e}_{j}\right\rangle .
$$

4) $G_{t}^{i}$, the level integrals for the state $\boldsymbol{e}_{i}$, is

$$
G_{t}^{i}=\int_{0}^{t}\left\langle X_{u}, \boldsymbol{e}_{i}\right\rangle d N_{u} .
$$

Using Bayes' Theorem, if $H=\left\{H_{t}, 0 \leq t\right\}$ is any $\mathcal{G}$ adapted process

$$
E\left[H_{t} \mid \mathcal{Y}_{0, t}\right]=\frac{E^{\dagger}\left[\Lambda_{0, t} H_{t} \mid \mathcal{Y}_{0, t}\right]}{E^{\dagger}\left[\Lambda_{0, t} \mid \mathcal{Y}_{0, t}\right]}
$$

Here, $E^{\dagger}[\cdot]$ denotes an expectation taken under the measure $P^{\dagger}$. Write

$$
\sigma\left(H_{t}\right)=E^{\dagger}\left[\Lambda_{0, t} H_{t} \mid \mathcal{Y}_{0, t}\right] .
$$

Indexing the sequence of passes of the EM algorithm by $\tau=$ $1,2,3 \ldots$, the update formulas for the parameter estimates are as follows:

$$
\left[\widehat{A}_{\tau+1}\right]_{(i, j)}=\frac{E\left[N_{T}^{(j, i)} \mid \mathcal{Y}_{0, T}\right]}{E\left[J_{T}^{i} \mid \mathcal{Y}_{0, T}\right]}=\frac{\sigma\left(N_{T}^{i, j}\right)}{\sigma\left(J_{T}^{i}\right)}
$$

and

$$
\left\langle\widehat{\lambda}_{\tau+1}, \boldsymbol{e}_{i}\right\rangle=\frac{E\left[G_{T}^{i} \mid \mathcal{Y}_{0, T}\right]}{E\left[J_{T}^{i} \mid \mathcal{Y}_{0, T}\right]}=\frac{\sigma\left(G_{T}^{i}\right)}{\sigma\left(J_{T}^{i}\right)}
$$

The conditional expectations in equations (3.20) and (3.21) are computed using the previous (at pass $\tau$ ) parameter estimates for $A$ and $\lambda$.

The updates for $\left[\hat{A}_{k}\right]_{(i, j)}$ and $\left\langle\hat{\lambda}_{k}, \boldsymbol{e}_{i}\right\rangle$ are computed by evaluating the expectations in (3.20) and (3.21), respectively. However, it is, in general, not possible to compute recursive dynamics for the processes $J^{i}, N^{(j, i)}$, and $G^{i}$. It is, however, possible to compute dynamics for the associated product quantities $\sigma\left(J_{t}^{i} X_{t}\right), \sigma\left(N_{t}^{(j, i)} X_{t}\right)$, and $\sigma\left(G_{t}^{i} X_{t}\right)$, where, for example,

$$
\sigma\left(G_{t}^{i} X_{t}\right)=E^{\dagger}\left[\Lambda_{t} G_{t}^{i} X_{t} \mid \mathcal{Y}_{t}\right] \in \mathbb{R}^{n} .
$$

The fundamental idea behind the filter-based EM algorithm is to compute recursive filters for quantities such as (3.22), then marginalize the state variable $X$ to evaluate the estimators given by (3.20) and (3.21). We now give recursive filters to estimate, respectively, the product quantities $J^{i} X, N^{(j, i)} X$, and $G^{i} X$.

Theorem 1: The vector-valued process $\sigma\left(J^{i} X\right) \in \mathbb{R}^{n}$ satisfies the stochastic integral equation

$$
\begin{aligned}
\sigma\left(J_{t}^{i} X_{t}\right)= & \int_{0}^{t} A \sigma\left(J_{u}^{i} X_{u}\right) d u+\int_{0}^{t}\left\langle q_{u}, \boldsymbol{e}_{i}\right\rangle d u \boldsymbol{e}_{i} \\
& +\int_{0}^{t} \operatorname{diag}\left\{\left\langle\lambda, \boldsymbol{e}_{\ell}\right\rangle-1\right\} \sigma\left(J_{u-}^{i} X_{u-}\right)\left(d N_{u}-d u\right) .
\end{aligned}
$$

Theoremm 2: The vector-valued process $\sigma\left(N^{(j, i)} X\right) \in \mathbb{R}^{n}$ satisfies the stochastic integral equation

168 169

$$
\begin{aligned}
\sigma\left(N_{t}^{(j, i)} X_{t}\right)= & \int_{0}^{t} A \sigma\left(N_{u}^{(j, i)} X_{u}\right) d u \\
& +\int_{0}^{t}\left\langle q_{u}, \boldsymbol{e}_{i}\right\rangle\left\langle A \boldsymbol{e}_{i}, \boldsymbol{e}_{j}\right\rangle d u \boldsymbol{e}_{j} \\
& \left.+\int_{0}^{t} \operatorname{diag}\left\{\left\langle\lambda, \boldsymbol{e}_{\ell}\right\rangle-1\right\rangle\right\} \\
& \times \sigma\left(N_{u-}^{(j, i)} X_{u-}\right)\left(d N_{u}-d u\right) .
\end{aligned}
$$

Here, $\sigma\left(N_{0}^{(j, i)} X_{0}\right)=0$ and $q$ is the solution of (3.12).

Theorem 3: The vector-valued process $\sigma\left(G^{i} X\right) \in \mathbb{R}^{n}$ satisfies the stochastic integral equation

$$
\begin{aligned}
\sigma\left(G_{t}^{i} X_{t}\right)= & \int_{0}^{t} A \sigma\left(G_{u}^{i} X_{u}\right) d u \\
& +\int_{0}^{t}\left\langle q_{u-}, \boldsymbol{e}_{i}\right\rangle\left\langle\lambda, \boldsymbol{e}_{i}\right\rangle d N_{u} \boldsymbol{e}_{i} \\
& +\int_{0}^{t} \operatorname{diag}\left\{\left\langle\lambda, \boldsymbol{e}_{\ell}\right\rangle-1\right\} \sigma\left(G_{u-}^{i} X_{u-}\right)\left(d N_{u}-d u\right) .
\end{aligned}
$$

Here, $\sigma\left(G_{0}^{i} X_{0}\right)=0$ and $q$ is the solution of (3.12).

A proof of Theorem 3 is given in the Appendix. Theorems 1174 and 2 can be readily proven by similar means. By using the solu- 175 tions of (3.23), (3.24), and (3.25), the updates for the parameter estimates are given by

$$
\left[\widehat{A}_{\tau+1}\right]_{(i, j)}=\frac{\left\langle\sigma\left(N_{T}^{(j, i)} X_{T}\right), \mathbf{1}\right\rangle}{\left\langle\sigma\left(J_{T}^{i} X_{T}\right), \mathbf{1}\right\rangle}
$$

and

$$
\left\langle\widehat{\lambda}_{\tau+1}, \boldsymbol{e}_{i}\right\rangle=\frac{\left\langle\sigma\left(G_{t}^{i} X_{T}\right), \mathbf{1}\right\rangle}{\left\langle\sigma\left(J_{T}^{i} X_{T}\right), \mathbf{1}\right\rangle} .
$$

\section{Robust State Estimation Filters}

Each of the dynamics given by (3.23)-(3.25) contain stochastic Lebesgue-Stieltjes integral terms. These stochastic integrals, with respect to the observation process $N$, can be eliminated by using a version of a gauge transformation due to Clark [1]. Consider the diagonal matrix

$$
\Gamma_{t} \triangleq \operatorname{diag}\left\{\gamma_{t}^{i}\right\} \in \mathbb{R}^{n \times n} .
$$

Here, $\gamma_{t}^{i} \triangleq \exp \left\{\left(1-\left\langle\lambda, \boldsymbol{e}_{i}\right\rangle\right) t\right\}\left\langle\lambda, \boldsymbol{e}_{i}\right\rangle^{N_{t}}$ with $\gamma_{0}^{i}=0$. Note that the matrix $\Gamma_{t}^{-1}$ is nonsingular. Using the Itô rule, one can show that

$$
\begin{aligned}
\Gamma_{t}^{-1}= & \int_{0}^{t} \operatorname{diag}\left\{\left\langle\lambda, \boldsymbol{e}_{\ell}\right\rangle-1\right\} \Gamma_{u}^{-1} d u \\
& +\int_{0}^{t} \Gamma_{u-}^{-1} \operatorname{diag}\left\{\left\langle\lambda, \boldsymbol{e}_{\ell}\right\rangle^{-1}-1\right\} d N_{u} .
\end{aligned}
$$




$$
\begin{aligned}
\Gamma_{t}^{-1} \sigma\left(G_{t}^{i} X_{t}\right)= & \int_{0}^{t} \Gamma_{u}^{-1} A \Gamma_{u} \bar{\sigma}\left(G_{u}^{i} X_{u}\right) d u \\
& +\int_{0}^{t}\left\langle\bar{q}_{u}, \boldsymbol{e}_{i}\right\rangle\left\langle\lambda, \boldsymbol{e}_{i}\right\rangle d N_{u} \boldsymbol{e}_{i} \\
& +\int_{0}^{t} \operatorname{diag}\left\{\left\langle\lambda, \boldsymbol{e}_{\ell}\right\rangle-1\right\} \bar{\sigma}\left(G_{u-}^{i} X_{u-}\right)\left(d N_{u}-d u\right) \\
& +\int_{0}^{t} \operatorname{diag}\left\{\left\langle\lambda, \boldsymbol{e}_{\ell}\right\rangle-1\right\} \bar{\sigma}\left(G_{u}^{i} X_{u}\right) d u \\
& +\int_{0}^{t} \operatorname{diag}\left\{\left\langle\lambda, \boldsymbol{e}_{\ell}\right\rangle^{-1}-1\right\} \bar{\sigma}\left(G_{u-}^{i} X_{u-}\right) d N_{u} \\
& +\int_{0}^{t} \operatorname{diag}\left\{\left\langle\lambda, \boldsymbol{e}_{\ell}\right\rangle^{-1}-1\right\}\left\langle\bar{q}_{u}, \boldsymbol{e}_{i}\right\rangle\left\langle\lambda, \boldsymbol{e}_{i}\right\rangle d N_{u} \boldsymbol{e}_{i} \\
& +\int_{0}^{t} \operatorname{diag}\left\{\left\langle\lambda, \boldsymbol{e}_{\ell}\right\rangle^{-1}-1\right\} \\
& \times \operatorname{diag}\left\{\left\langle\lambda, \boldsymbol{e}_{\ell}\right\rangle^{-1}-1\right\} \bar{\sigma}\left(G_{u-}^{i} X_{u-}\right) d N_{u} .
\end{aligned}
$$

Finally, our dynamics for $\bar{\sigma}\left(G_{t}^{i} X_{t}\right)$ read

$$
\begin{aligned}
\bar{\sigma}\left(G_{t}^{i} X_{t}\right)= & \int_{0}^{t} \Gamma_{u}^{-1} A \Gamma_{u} \bar{\sigma}\left(G_{u}^{i} X_{u}\right) d u \\
& +\left\langle\bar{q}_{t}, \boldsymbol{e}_{i}\right\rangle N_{t} \boldsymbol{e}_{i}-\int_{0}^{t} N_{u}\left\langle d \bar{q}_{u}, \boldsymbol{e}_{i}\right\rangle \boldsymbol{e}_{i} .
\end{aligned}
$$

Similarly, one can apply the product rule to compute process dy- 202 namics for the quantities $\bar{\sigma}\left(J_{t}^{i} X_{t}\right)$ and $\bar{\sigma}\left(N_{t}^{(j, i)} X_{t}\right)$. The results 203 of these calculations are, respectively,

$$
\bar{\sigma}\left(J_{t}^{i} X_{t}\right)=\int_{0}^{t} \Gamma_{u}^{-1} A \Gamma_{u} \bar{\sigma}\left(J_{u}^{i} X_{u}\right) d u+\int_{0}^{t}\left\langle\bar{q}_{u}, \boldsymbol{e}_{i}\right\rangle d u \boldsymbol{e}_{i}
$$

and

$$
\begin{aligned}
\bar{\sigma}\left(N_{t}^{(j, i)} X_{t}\right)= & \int_{0}^{t} \Gamma_{u}^{-1} A \Gamma_{u} \bar{\sigma}\left(N_{u}^{(j, i)} X_{u}\right) d u \\
& +\int_{0}^{t}\left\langle\bar{q}_{u}, \boldsymbol{e}_{i}\right\rangle\left\langle A \boldsymbol{e}_{i}, \boldsymbol{e}_{j}\right\rangle d u \boldsymbol{e}_{j} .
\end{aligned}
$$

\section{Discrete-Time Filters}

For all time discretizations, we will consider a partition on an 207 interval $[0, T]$ and write

$$
\Pi_{[0, T]}^{(K)} \triangleq\left\{0=t_{0}, t_{1}, \ldots, t_{K}=T\right\} .
$$

Here, the partition is strict, that is, $t_{0}<t_{1}<\cdots<t_{K}=T$. To 209 denote the mesh of the partition, we write

$$
\left\|\Pi_{[0, T]}^{(K)}\right\|=\max _{1 \leq k \leq K}\left\{t_{k}-t_{k-1}\right\} .
$$

For brevity, we shall use the notation $\xi_{k} \triangleq \xi_{t_{k}}$, where $\xi_{k}$ denotes 211 a process $\xi$ at a time point $t_{k}$. Further, we write $\Delta_{(k-1, k)}=212$ $t_{k}-t_{k-1}$. Approximating the integral in (3.30), we get

$$
\bar{q}_{t_{k}} \approx \bar{q}_{t_{k-1}}+\Gamma_{t_{k-1}}^{-1} A \Gamma_{t_{k-1}} \bar{q}_{t_{k-1}} \Delta_{(k-1, k)}
$$

so

$$
q_{t_{k}}=\Gamma_{t_{k}} \bar{q}_{t_{k}} \approx \Gamma_{t_{k}} \Gamma_{t_{k-1}}^{-1}\left[\mathbf{I}+\Delta_{(k-1, k)} A\right] q_{t_{k-1}} .
$$

This suggests the recursion

$$
\widehat{q}_{k} \triangleq \Gamma_{k} \Gamma_{k-1}^{-1}\left[\mathbf{I}+\Delta_{(k-1, k)} A\right] \widehat{q}_{k-1} .
$$

Here, $\widehat{q}$ denotes an estimate of the unnormalized probability 216 generated by the suboptimal discrete-time recursion at (3.43). 217

Remark 1: An important feature of the filter formulation at 218 (3.44) is that the sampling interval or $\Delta_{(k-1, k)}$ can be chosen 219 to ensure a certain type of numerical stability. Here, numerical 220 stability is taken to mean $\left\langle q, \boldsymbol{e}_{i}\right\rangle \geq 0$ for all $i \in\{1,2, \ldots, n\} . \quad 221$ The details of this property are given in [16].

Writing the dynamics given by (3.37) recursively at sampling 223 instants $t_{k}$ and $t_{k-1}$, we get

$$
\begin{aligned}
\bar{\sigma}\left(G_{t_{k}}^{i} X_{t_{k}}\right)= & \bar{\sigma}\left(G_{t_{k-1}}^{i} X_{t_{k-1}}\right) \\
& +\int_{t_{k-1}}^{t_{k}} \Gamma_{u}^{-1} A \Gamma_{u} \bar{\sigma}\left(G_{u}^{i} X_{u}\right) d u
\end{aligned}
$$

$$
\int_{0}^{t}\left\langle\bar{q}_{u}, \boldsymbol{e}_{i}\right\rangle d N_{u} \boldsymbol{e}_{i}=\left\langle\bar{q}_{t}, \boldsymbol{e}_{i}\right\rangle N_{t}-\int_{0}^{t} N_{u}\left\langle d \bar{q}_{u}, \boldsymbol{e}_{i}\right\rangle .
$$




$$
\begin{aligned}
& +\left\langle\bar{q}_{t_{k}}, \boldsymbol{e}_{i}\right\rangle N_{t_{k}} \boldsymbol{e}_{i}-\left\langle\bar{q}_{t_{k-1}}, \boldsymbol{e}_{i}\right\rangle N_{t_{k-1}} \boldsymbol{e}_{i} \\
& -\int_{t_{k-1}}^{t_{k}} N_{u}\left\langle d \bar{q}_{u}, \boldsymbol{e}_{i}\right\rangle \boldsymbol{e}_{i} .
\end{aligned}
$$

Making an Euler-Maruyama ${ }^{1}$ approximation, we have

$$
\begin{aligned}
\int_{t_{k-1}}^{t_{k}} N_{u}\left\langle d \bar{q}_{u}, \boldsymbol{e}_{i}\right\rangle \boldsymbol{e}_{i} & =\int_{t_{k-1}}^{t_{k}} N_{u}\left\langle\Gamma_{u}^{-1} A \Gamma_{u} \bar{q}_{u} d u, \boldsymbol{e}_{i}\right\rangle \boldsymbol{e}_{i} \\
& \approx N_{t_{k-1}} \Gamma_{t_{k-1}}^{-1}\left\langle A q_{t_{k-1}} \boldsymbol{e}_{i}\right\rangle \Delta_{(k-1, k)} \boldsymbol{e}_{i}
\end{aligned}
$$

and with some algebraic manipulation

$$
\begin{aligned}
\left\langle\bar{q}_{t_{k}},\right. & \left.\boldsymbol{e}_{i}\right\rangle N_{t_{k}} \boldsymbol{e}_{i}-\left\langle\bar{q}_{t_{k-1}}, \boldsymbol{e}_{i}\right\rangle N_{t_{k-1}} \boldsymbol{e}_{i} \\
= & \Gamma_{t_{k-1}}^{-1}\left\langle q_{t_{k-1}}, \boldsymbol{e}_{i}\right\rangle\left(N_{t_{k}}-N_{t_{k-1}}\right) \boldsymbol{e}_{i} \\
& \quad+\Delta_{(k-1, k)} \Gamma_{t_{k-1}}^{-1}\left\langle A q_{t_{k-1}}, \boldsymbol{e}_{i}\right\rangle N_{t_{k}} \boldsymbol{e}_{i}
\end{aligned}
$$

227

Now, by multiplying both sides of (3.48) on the left-hand side by the matrix $\Gamma_{k}$, we get

$$
\begin{aligned}
\sigma\left(G_{k}^{i} X_{k}\right) \approx & \Gamma_{k} \Gamma_{k-1}^{-1} \sigma\left(G_{k-1}^{i} X_{k-1}\right) \\
& +\Gamma_{k} \Gamma_{k-1}^{-1} A \sigma\left(G_{k-1}^{i} X_{k-1}\right) \Delta_{(k-1, k)} \\
& +\Gamma_{k} \Gamma_{k-1}^{-1}\left\langle\widehat{q}_{k-1}, \boldsymbol{e}_{i}\right\rangle\left(N_{k}-N_{k-1}\right) \boldsymbol{e}_{i} \\
& +\Gamma_{k} \Gamma_{k-1}^{-1}\left\langle A \widehat{q}_{k-1}, \boldsymbol{e}_{i}\right\rangle\left(N_{k}-N_{k-1}\right) \boldsymbol{e}_{i} .
\end{aligned}
$$

230

After similar calculations, the remaining discretized filters read

$$
\begin{aligned}
\widehat{\sigma}\left(N_{k}^{(j, i)} X_{k}\right)= & \Gamma_{k} \Gamma_{k-1}^{-1}\left[\mathbf{I}+\Delta_{(k-1, k)} A\right] \widehat{\sigma}\left(N_{k-1}^{(j, i)} X_{k-1}\right) \\
& +\Gamma_{k} \Gamma_{k-1}^{-1}\left\langle\widehat{q}_{k-1}, \boldsymbol{e}_{i}\right\rangle\left\langle A \boldsymbol{e}_{i}, \boldsymbol{e}_{j}\right\rangle \Delta_{(k-1, k)} \boldsymbol{e}_{i}
\end{aligned}
$$

$$
\begin{aligned}
\widehat{\sigma}\left(J_{k}^{i} X_{k}\right)= & \Gamma_{k} \Gamma_{k-1}^{-1}\left[\mathbf{I}+\Delta_{(k-1, k)} A\right] \widehat{\sigma}\left(J_{k-1}^{i} X_{k-1}\right) \\
& +\Gamma_{k} \Gamma_{k-1}^{-1}\left\langle\widehat{q}_{k-1}, \boldsymbol{e}_{i}\right\rangle \boldsymbol{e}_{i} .
\end{aligned}
$$

\footnotetext{
${ }^{1}$ We will use this particular approximation throughout this paper, however, other approximations could also be used.
}

\section{E. Discrete-Time Filter-Based EM Algorithm}

Summarizing the results from the previous sections, our filterbased EM algorithm reads

Initialization $\forall(i, j) \in\{(1,1),(1,2), \ldots,(n, n)\}$, Choose $\left[\widehat{A}_{0}\right]_{(i, j)}$, for each $i \in\{1,2, \ldots, n\}$ choose $\left\langle\widehat{\lambda}, \boldsymbol{e}_{i}\right\rangle$.

Step $1 \quad$ Using (3.26) and (3.27), compute the MLEs, $\left[\widehat{A}_{\tau+1}\right]_{i, j}$ and $\widehat{\lambda}_{\tau+1}$.

Step 2 Decide to stop or continue from step 2.

\section{SMOOTHER-BASEd EM ALgORITHM FOR MMPPS}

In many implementations of the EM algorithm, for example, 237 [24] and [29], the expectation step is completed with smoothed 238 rather than (online) filtered estimates. Typically, the smoothing 239 scheme used is the so-called "fixed interval smoother." Com- 240 puting smoothing schemes for MMPPs can be particularly diffi- 241 cult [23], [26]. One source of this difficulty is the task of devel- 242 oping backwards dynamics. This task usually leads to construct- 243 ing stochastic integrals evolving backward in time. However, the 244 approach we use to develop smoothing algorithms completely 245 avoids these difficulties. To compute our smoothers we exploit 246 a special identity between forward and backward robust dynam- 247 ics, and as a consequence, do not need to consider the backward 248 stochastic integration at all.

\section{A. Smoothed State Estimation for the Process $X$}

We first briefly recall the state estimation MMPP smoother 251 presented in [14]. For a smoothed estimate for the process $X \in 252$ $\mathbb{R}^{n}$, we wish to evaluate the expectation $E\left[X_{t} \mid \mathcal{Y}_{0, T}\right]$, where 253 $0 \leq t \leq T$. By Bayes' rule [3], we have

$$
E\left[X_{t} \mid \mathcal{Y}_{0, T}\right]=\frac{E^{\dagger}\left[\Lambda_{0, T} X_{t} \mid \mathcal{Y}_{0, T}\right]}{E^{\dagger}\left[\Lambda_{0, T} \mid \mathcal{Y}_{0, T}\right]} .
$$

Consider the numerator of (4.53)

$$
\begin{aligned}
r_{t} & \triangleq E^{\dagger}\left[\Lambda_{0, T} X_{t} \mid \mathcal{Y}_{0, T}\right] \\
& =E^{\dagger}\left[\Lambda_{0, t} \Lambda_{t, T} X_{t} \mid \mathcal{Y}_{0, T}\right] \\
& =E^{\dagger}\left[E^{\dagger}\left[\Lambda_{0, t} \Lambda_{t, T} X_{t} \mid \mathcal{Y}_{0, T} \vee \mathcal{F}_{t}\right] \mid \mathcal{Y}_{0, T}\right] \\
& =E^{\dagger}\left[\Lambda_{0, t} X_{t} E^{\dagger}\left[\Lambda_{t, T}\left|\mathcal{Y}_{0, T} \vee \mathcal{F}_{t}\right| \mathcal{Y}_{0, T}\right] .\right.
\end{aligned}
$$

Under the measure $P^{\dagger}, X$ is a Markov process, so the inner 256 expectation in the previous line of (4.54) is

$$
E^{\dagger}\left[\Lambda_{t, T} \mid \mathcal{Y}_{0, T} \vee \mathcal{F}_{t}\right]=E^{\dagger}\left[\Lambda_{t, T} \mid \mathcal{Y}_{0, T} \vee \sigma\left\{X_{t}\right\}\right]
$$

Write

$$
v_{t, T}^{i} \triangleq E^{\dagger}\left[\Lambda_{t, T} \mid \mathcal{Y}_{0, T} \text { and } X_{t}=\boldsymbol{e}_{i}\right] .
$$

Omitting further calculations, it can be shown [14] that

$$
\begin{aligned}
r_{t}= & \left\langle q_{t}, \boldsymbol{e}_{1}\right\rangle\left\langle v_{t, T}, \boldsymbol{e}_{1}\right\rangle \boldsymbol{e}_{1}+\left\langle q_{t}, \boldsymbol{e}_{2}\right\rangle\left\langle v_{t, T}, \boldsymbol{e}_{2}\right\rangle \boldsymbol{e}_{2}+\cdots \\
& +\left\langle q_{t}, \boldsymbol{e}_{m}\right\rangle\left\langle v_{t, T}, \boldsymbol{e}_{n}\right\rangle \boldsymbol{e}_{n} \in \mathbb{R}^{n} .
\end{aligned}
$$

The normalized smoothed-state estimate of $X$ is then

$$
E\left[X_{t} \mid \mathcal{Y}_{0, t}\right]=\frac{r_{t}}{\left\langle r_{t}, \mathbf{1}\right\rangle} .
$$


Note that

$$
\begin{aligned}
\left\langle r_{t}, \mathbf{1}\right\rangle & =\left\langle q_{t}, v_{t, T}\right\rangle \\
& =E^{\dagger}\left[\Lambda_{0, T}\left\langle X_{t}, \mathbf{1}\right\rangle \mid \mathcal{Y}_{0, T}\right] \\
& =E^{\dagger}\left[\Lambda_{0, T} \mid \mathcal{Y}_{0, T}\right]
\end{aligned}
$$

262

$\left\langle\bar{q}_{t}, \bar{v}_{t, T}\right\rangle=\left\langle\Gamma_{t}^{-1} q_{t}, \Gamma_{t} v_{t, T}\right\rangle=\left\langle q_{t}, v_{t, T}\right\rangle$,

for all $t \in[0, T]$.

is independent of $t$. Therefore

$$
\begin{aligned}
\frac{d}{d t}\left\langle r_{t}, \mathbf{1}\right\rangle & =\frac{d}{d t}\left\langle q_{t}, v_{t, T}\right\rangle \\
& =\left\langle d \bar{q}_{t}, \bar{v}_{t, T}\right\rangle+\left\langle\bar{q}_{t}, d \bar{v}_{t, T}\right\rangle \\
& =\left\langle\Gamma_{t}^{-1} A \Gamma_{t} \bar{q}_{t}, \bar{v}_{t, T}\right\rangle+\left\langle\bar{q}_{t}, d \bar{v}_{t, T}\right\rangle \\
& =0 .
\end{aligned}
$$

The vector $v_{t, T}=\left(\left\langle v_{t, T}, \boldsymbol{e}_{1}\right\rangle,\left\langle v_{t, T}, \boldsymbol{e}_{2}\right\rangle, \ldots,\left\langle v_{t, T}, \boldsymbol{e}_{n}\right\rangle\right)$ incorporates the extra information obtained from the observations between $t$ and $T$. Computing dynamics for $v$ can be difficult [18], [19]. However, by exploiting a special identity between the forward dynamics and the corresponding backward, process $\bar{v}$, one can directly compute robust dynamics for the process $v$. What we must do is consider the process $\bar{v}$, such that the following identity holds

That is, $\bar{v}_{t, T} \triangleq \Gamma_{t} v_{t, T}$. Using (4.60), one can show that

$$
\frac{d \bar{v}_{t, T}}{d t}=-\Gamma_{t} A^{\prime} \Gamma_{t}^{-1} \bar{v}_{t, T}, \quad \bar{v}_{T, T}=v_{T, T}=\mathbf{1}
$$

so that

$$
\bar{v}_{t, T}=\mathbf{1}+\int_{t}^{T} \Gamma_{u} A^{\prime} \Gamma_{u}^{-1} \bar{v}_{t, T} d u .
$$

Further, using the time discretization of (3.40)

$$
\begin{aligned}
\bar{v}_{k-1, T} & =\bar{v}_{k, T}+\int_{t_{k-1}}^{t_{k}} \Gamma_{u} A^{\prime} \Gamma_{u}^{-1} \bar{v}_{u, T} d u \\
& \approx \bar{v}_{k, T}+\Gamma_{k} A^{\prime} \Gamma_{k}^{-1} \bar{v}_{k, T} \Delta_{(k-1, k)},
\end{aligned}
$$

so, our suboptimal estimator $\widehat{v} \approx v$ has dynamics

$$
\widehat{v}_{k-1, T} \triangleq \Gamma_{k-1}^{-1} \Gamma_{k}\left[\boldsymbol{I}+\Delta_{(k-1, k)} A^{\prime}\right] \widehat{v}_{k, T} .
$$

\section{B. Smoothers for the Quantities $N_{t}^{i}, J_{t}^{i}$, and $G_{t}^{i}$}

Following the same strategy as before, we consider the identity

$$
\begin{aligned}
\left\langle\sigma\left(G_{t}^{i} X_{t}\right), v_{t, T}\right\rangle & =\left\langle\Gamma_{t}^{-1} \sigma\left(G_{t}^{i} X_{t}\right), \Gamma_{t} v_{t, T}\right\rangle \\
& =\left\langle\bar{\sigma}\left(G_{t}^{i} X_{t}\right), \bar{v}_{t, T}\right\rangle .
\end{aligned}
$$

Now, define

$$
\widetilde{\sigma}\left(G_{t}^{i} X_{t}\right) \triangleq \bar{\sigma}\left(G_{t}^{i} X_{t}\right)-\left\langle\bar{q}_{t}, \boldsymbol{e}_{i}\right\rangle N_{t} \boldsymbol{e}_{i}
$$

Then

$$
\begin{aligned}
d \tilde{\sigma}\left(G_{t}^{i} X_{t}\right)= & \Gamma_{t}^{-1} A \Gamma_{t} \bar{\sigma}\left(G_{t}^{i} X_{t}\right) d t \\
& -N_{t}\left\langle\Gamma_{t}^{-1} A \Gamma_{t} \bar{q}_{t}, \boldsymbol{e}_{i}\right\rangle \boldsymbol{e}_{i} d t
\end{aligned}
$$

Now,

$$
\left\langle\bar{\sigma}\left(G_{t}^{i} X_{t}\right), \bar{v}_{t, T}\right\rangle=\left\langle\widetilde{\sigma}\left(G_{t}^{i} X_{t}\right), \bar{v}_{t, T}\right\rangle+N_{t}\left\langle\bar{q}_{t}, \boldsymbol{e}_{i}\right\rangle\left\langle\bar{v}_{t, T}, \boldsymbol{e}_{i}\right\rangle
$$

and

$$
N_{t}\left\langle\bar{q}_{t}, \boldsymbol{e}_{i}\right\rangle\left\langle\bar{v}_{t, T}, \boldsymbol{e}_{i}\right\rangle=N_{t}\left\langle q_{t}, \boldsymbol{e}_{i}\right\rangle\left\langle v_{t, T}, \boldsymbol{e}_{i}\right\rangle .
$$

From the dynamics of $\widetilde{\sigma}\left(G_{t}^{i} X_{t}\right)$, we have

$$
\begin{aligned}
d\left\langle\tilde{\sigma}\left(G_{t}^{i} X_{t}\right), \bar{v}_{t, T}\right\rangle & \\
= & \left\langle\Gamma_{t}^{-1} A \Gamma_{t} \bar{\sigma}\left(G_{t}^{i} X_{t}\right), \bar{v}_{t, T}\right\rangle d t \\
& -N_{t}\left\langle\Gamma_{t}^{-1} A \Gamma_{t} \bar{q}_{t}, \boldsymbol{e}_{i}\right\rangle\left\langle\boldsymbol{e}_{i}, \bar{v}_{t, T}\right\rangle d t \\
& -\left\langle\widetilde{\sigma}\left(G_{t}^{i} X_{t}\right), \Gamma_{t} A \Gamma_{t}^{-1} \bar{v}_{t, T}\right\rangle d t \\
= & \left\langle\Gamma_{t}^{-1} A \Gamma_{t} \bar{\sigma}\left(G_{t}^{i} X_{t}\right), \bar{v}_{t, T}\right\rangle d t \\
& -N_{t}\left\langle\Gamma_{t}^{-1} A \Gamma_{t} \bar{q}_{t}, \boldsymbol{e}_{i}\right\rangle\left\langle\boldsymbol{e}_{i}, \bar{v}_{t, T}\right\rangle d t \\
& -\left\langle\bar{\sigma}\left(G_{t}^{i} X_{t}\right)-\left\langle\bar{q}_{t}, \boldsymbol{e}_{i}\right\rangle N_{t} \boldsymbol{e}_{i}, \Gamma_{t} A^{\prime} \Gamma_{t}^{-1} \bar{v}_{t, T}\right\rangle d t \\
= & -N_{t}\left\langle\Gamma_{t}^{-1} A \Gamma_{t} \bar{q}_{t}, \boldsymbol{e}_{i}\right\rangle\left\langle\boldsymbol{e}_{i}, \bar{v}_{t, T}\right\rangle d t \\
& +N_{t}\left\langle\bar{q}_{t}, \boldsymbol{e}_{i}\right\rangle\left\langle\Gamma_{t}^{-1} A \Gamma_{t} \boldsymbol{e}_{i}, \bar{v}_{t, T}\right\rangle d t
\end{aligned}
$$

i.e.,

\section{$\left\langle\widetilde{\sigma}\left(G_{T}^{i} X_{T}\right), \bar{v}_{T, T}\right\rangle$}

$$
\begin{aligned}
= & -\int_{0}^{T} N_{u}\left\langle\Gamma_{u}^{-1} A \Gamma_{u} \bar{q}_{u}, \boldsymbol{e}_{i}\right\rangle\left\langle\boldsymbol{e}_{i}, \bar{v}_{u, T}\right\rangle d u \\
& +\int_{0}^{T} N_{u}\left\langle\bar{q}_{u}, \boldsymbol{e}_{i}\right\rangle\left\langle\Gamma_{u}^{-1} A \Gamma_{u} \boldsymbol{e}_{i}, \bar{v}_{u, T}\right\rangle d u .
\end{aligned}
$$

Therefore,

$$
\begin{aligned}
\left\langle\sigma\left(G_{T}^{i} X_{T}\right), v_{T, T}\right\rangle= & \left\langle\bar{\sigma}\left(G_{T}^{i} X_{T}\right), \bar{v}_{T}\right\rangle \\
= & \left\langle\widetilde{\sigma}\left(G_{T}^{i} X_{T}\right), \bar{v}_{T}\right\rangle+N_{T}\left\langle\bar{q}_{T}, \boldsymbol{e}_{i}\right\rangle\left\langle\bar{v}_{T}, \boldsymbol{e}_{i}\right\rangle \\
= & -\int_{0}^{T} N_{u}\left\langle\Gamma_{u}^{-1} A \Gamma_{u} \bar{q}_{u}, \boldsymbol{e}_{i}\right\rangle\left\langle\boldsymbol{e}_{i}, \bar{v}_{u, T}\right\rangle d u \\
& +\int_{0}^{T} N_{u}\left\langle\bar{q}_{u}, \boldsymbol{e}_{i}\right\rangle\left\langle\Gamma_{u}^{-1} A \Gamma_{u} \boldsymbol{e}_{i}, \bar{v}_{u, T}\right\rangle d u \\
& +N_{T}\left\langle q_{T}, \boldsymbol{e}_{i}\right\rangle\left\langle v_{T, T}, \boldsymbol{e}_{i}\right\rangle \\
= & -\int_{0}^{T} N_{u}\left\langle A q_{u}, \boldsymbol{e}_{i}\right\rangle\left\langle\boldsymbol{e}_{i}, v_{u, T}\right\rangle d u \\
& +\int_{0}^{T} N_{u}\left\langle q_{u}, \boldsymbol{e}_{i}\right\rangle\left\langle\boldsymbol{e}_{i}, A^{\prime} v_{u, T}\right\rangle d u \\
& +N_{T}\left\langle q_{T}, \boldsymbol{e}_{i}\right\rangle\left\langle v_{T, T}, \boldsymbol{e}_{i}\right\rangle .
\end{aligned}
$$

By using similar calculations, one can also show that

$$
\left\langle\bar{\sigma}\left(J_{T}^{i} X_{T}\right), \bar{v}_{T, T}\right\rangle=\int_{0}^{T}\left\langle q_{u}, \boldsymbol{e}_{i}\right\rangle\left\langle v_{u, T}, \boldsymbol{e}_{i}\right\rangle d t
$$

and

$$
\left\langle\bar{\sigma}\left(N_{T}^{(j, i)} X_{T}\right), \bar{v}_{T, T}\right\rangle=\int_{0}^{T}\left\langle A \boldsymbol{e}_{i}, \boldsymbol{e}_{j}\right\rangle\left\langle q_{u}, \boldsymbol{e}_{i}\right\rangle\left\langle v_{u, T}, \boldsymbol{e}_{j}\right\rangle d u
$$




\section{Smoother-Based EM Algorithm}

Recalling (3.20) and (3.21), our smoother-based update equations are

$$
\left[\widehat{A}_{\tau+1}\right]_{(i, j)}=\left[\widehat{A}_{\tau}\right]_{(i, j)} \frac{\int_{0}^{T}\left\langle q_{u}, \boldsymbol{e}_{i}\right\rangle\left\langle v_{u, T}, \boldsymbol{e}_{j}\right\rangle d u}{\int_{0}^{T}\left\langle q_{u}, \boldsymbol{e}_{i}\right\rangle\left\langle v_{u, T}, \boldsymbol{e}_{i}\right\rangle d u}
$$

and

$$
\begin{aligned}
\left\langle\widehat{\lambda}_{\tau+1}, \boldsymbol{e}_{i}\right\rangle= & \frac{\int_{0}^{T} N_{u}\left\langle q_{u}, \boldsymbol{e}_{i}\right\rangle\left\langle\boldsymbol{e}_{i}, A^{\prime} v_{u, T}\right\rangle d u}{\int_{0}^{T}\left\langle q_{u}, \boldsymbol{e}_{i}\right\rangle\left\langle v_{u, T}, \boldsymbol{e}_{i}\right\rangle d u} \\
& -\frac{\int_{0}^{T} N_{u}\left\langle A q_{u}, \boldsymbol{e}_{i}\right\rangle\left\langle\boldsymbol{e}_{i}, v_{u, T}\right\rangle d u}{\int_{0}^{T}\left\langle q_{u}, \boldsymbol{e}_{i}\right\rangle\left\langle v_{u, T}, \boldsymbol{e}_{i}\right\rangle d u} \\
& +\frac{N_{T}\left\langle q_{T}, \boldsymbol{e}_{i}\right\rangle\left\langle v_{T, T}, \boldsymbol{e}_{i}\right\rangle}{\int_{0}^{T}\left\langle q_{u}, \boldsymbol{e}_{i}\right\rangle\left\langle v_{u, T}, \boldsymbol{e}_{i}\right\rangle d u} .
\end{aligned}
$$

\section{DisCRETE-Time SMOOTHERS}

\section{A. Discrete-Time Smoother Formulas}

Suppose that one observes data on the set $[0, T]$ and parameter estimates are computed by using these data. Further, suppose one receives a subsequent observation data on the set $\left[T, T^{\prime}\right]$, where $T^{\prime}>T$. What we would like to do is incorporate the new data on $\left[T, T^{\prime}\right]$ so as to reestimate the model parameters, but without complete recalculation from the origin. To utilize the information on $\left[T, T^{\prime}\right]$, we consider a time discretization on the total interval $[0, T] \cup\left[T, T^{\prime}\right]$, that is,

$$
0=t_{0}<t_{1} \cdots<t_{K}=T<t_{0}^{\prime}<t_{1}^{\prime} \cdots<t_{\widetilde{K}}=T^{\prime} .
$$

Here, we denote this augmented partition by $\Pi_{[0, T]}^{(K)} \cup \Pi_{\left[T, T^{\prime}\right]}^{(\widetilde{K})}$, where $\widetilde{K} \in \mathbb{N}$ and

$$
\Pi_{\left[T, T^{\prime}\right]}^{(\widetilde{K})} \triangleq\left\{T=t_{0}^{\prime}, t_{1}^{\prime}, \ldots, t_{\widetilde{K}}^{\prime}=T^{\prime}\right\} .
$$

Recalling the discrete-time, (backward) recursion for the estimator $\widehat{v}$, we see

$$
\begin{aligned}
\widehat{v}_{k-1, T}= & \Gamma_{k-1}^{-1} \Gamma_{k}\left[\boldsymbol{I}+\Delta_{(k-1, k)} A^{\prime}\right] \Gamma_{k-1}^{-1} \Gamma_{k} \widehat{v}_{k, T} \\
= & \Gamma_{k-1}^{-1} \Gamma_{k}\left[\boldsymbol{I}+\Delta_{(k-1, k)} A^{\prime}\right] \Gamma_{k}^{-1} \Gamma_{k+1}\left[\boldsymbol{I}+\Delta_{(k, k+1)} A^{\prime}\right] \\
& \quad \ldots, \Gamma_{K-1}^{-1} \Gamma_{K}\left[\boldsymbol{I}+\Delta_{(k-1, k)} A^{\prime}\right] \widehat{v}_{T, T} .
\end{aligned}
$$

Recall here that $K=t_{K}=T$.

Write

$$
\begin{aligned}
\Psi_{k-1, T} \triangleq & \Gamma_{k-1}^{-1} \Gamma_{k}\left[\boldsymbol{I}+\Delta_{(k-1, k)} A^{\prime}\right] \Gamma_{k}^{-1} \Gamma_{k+1} \\
& \times\left[\boldsymbol{I}+\Delta_{(k, k+1)} A^{\prime}\right], \ldots \Gamma_{K-1}^{-1} \Gamma_{K}\left[\boldsymbol{I}+\Delta_{(k-1, k)} A^{\prime}\right] \\
\in & \mathbb{R}^{n \times n} .
\end{aligned}
$$

Further, for two epochs $T$ and $T^{\prime}$, where $T<T^{\prime}$, it follows that

$$
\Psi_{k-1, T^{\prime}}=\Psi_{k-1, T} \Psi_{T, T^{\prime}}, \quad k \in\{0,1,2, \ldots, T\} .
$$

At a boundary $T^{\prime}$, we set $\Psi_{T^{\prime}, T^{\prime}} \triangleq \operatorname{diag}\{1,1, \ldots, 1\} \in \mathbb{R}^{n \times n}$.

Remarks 2 : The transitivity property for $\Psi$ shown by equation (5.82) is critical in our development of data-recursive smoother update formulas.
Using the matrix $\Psi$, the backward recursion for $v_{k-1, T}$ may 312 be written in the following compact form:

$$
v_{k-1, T}=\Psi_{k-1, T} \mathbf{1}, \quad \mathbf{1}=(1,1, \ldots, 1)^{\prime} \in \mathbb{R}^{n} .
$$

Equation (5.82) and the transitivity property of $\Psi$ can be exploited to compute a data-recursive smoother, that is, a smoother that does not require complete recalculation from the origin upon the arrival of new observation data. Since $\Psi$ is an $n \times n$ matrix, it can be easily stored in memory. It is immediate from the dynamics at (5.82) that the boundary $T$, upon which $v_{k-1 . T}$ depends, is only "fixed" by the action of multiplication on the right-hand side by the vector 1 . To extend this boundary upon the arrival of subsequent data, the $n \times n$ matrix $\Psi_{k-1, T}$ can be recalled from memory and the updated quantity $v_{k-1, T^{\prime}}$ is calculated by the recursion

$$
v_{k-1, T^{\prime}}=\Psi_{k-1, T} \Psi_{T, T^{\prime}} \mathbf{1}, \quad \mathbf{1}=(1,1, \ldots, 1)^{\prime} \in \mathbb{R}^{n} .
$$

Consider, for example, the following smoothing problem. Suppose one first observes data on $[0, T]$ and computes the smoothed estimate $P\left(X_{t}=\boldsymbol{e}_{i} \mid \mathcal{Y}_{0, T}\right)$ for some $t \in[0, T]$. Using $\Psi$, this estimation can be written as

$$
\begin{aligned}
P\left(X_{t}=\boldsymbol{e}_{i} \mid \mathcal{Y}_{0, T}\right) & =\frac{\left\langle q_{t}, \boldsymbol{e}_{i}\right\rangle\left\langle v_{t, T}, \boldsymbol{e}_{i}\right\rangle}{\sum_{\ell=1}^{n}\left\langle q_{t}, \boldsymbol{e}_{\ell}\right\rangle\left\langle v_{t, T}, \boldsymbol{e}_{\ell}\right\rangle} \\
& =\frac{\left\langle q_{t}, \boldsymbol{e}_{i}\right\rangle\left(\boldsymbol{e}_{\ell}^{\prime} \Psi_{t, T}\right) \mathbf{1}}{\left\{\sum_{\ell=1}^{n}\left\langle q_{t}, \boldsymbol{e}_{\ell}\right\rangle\left(\boldsymbol{e}_{\ell}^{\prime} \Psi_{t, T}\right)\right\} \mathbf{1}} .
\end{aligned}
$$

Now, suppose subsequent data are received on $\left[T, T^{\prime}\right]$ and we wish to compute $P\left(X_{t}=\boldsymbol{e}_{i} \mid \mathcal{Y}_{0, T^{\prime}}\right)$. Using $\Psi_{T, T^{\prime}}$, this estimate may be computed by

$$
\begin{aligned}
P\left(X_{t}=\boldsymbol{e}_{i} \mid \mathcal{Y}_{0, T^{\prime}}\right) & =\frac{\left\langle q_{t}, \boldsymbol{e}_{i}\right\rangle\left\langle v_{t, T^{\prime}}, \boldsymbol{e}_{i}\right\rangle}{\sum_{\ell=1}^{n}\left\langle q_{t}, \boldsymbol{e}_{\ell}\right\rangle\left\langle v_{t, T^{\prime}}, \boldsymbol{e}_{\ell}\right\rangle} \\
& =\frac{\left\langle q_{t}, \boldsymbol{e}_{i}\right\rangle\left(\boldsymbol{e}_{\ell}^{\prime} \Psi_{t, T}\right) \Psi_{T}, T^{\prime} \mathbf{1}}{\left\{\sum_{\ell=1}^{n}\left\langle q_{t}, \boldsymbol{e}_{\ell}\right\rangle\left(\boldsymbol{e}_{\ell}^{\prime} \Psi_{t, T}\right)\right\} \Psi_{T, T^{\prime}} \mathbf{1}}
\end{aligned}
$$

Equation (5.85) shows that the smoother probability can be com- 332 puted without the recalculation of $v$ from the origin, provided 333 the $n \times n$ matrix $\Psi_{t, T}$ has been stored in memory.

\section{B. Discrete-Time Smoother-Based EM Algorithm}

To compute discrete-time approximations of update formulas (4.76) and (4.77), we approximate the integrals in these estimators by the Trapezoidal rule. These approximations can also be written in a data-recursive form. To approximate $\left[\widehat{A}_{\tau+1}\right]_{(i, j)}$ on the interval $[0, T]$, we write (5.86), as shown at the bottom the next page.

Similarly, (5.87) as shown at the bottom of the next page. 342

Consider again the scenario of new observation data and the 343 two time intervals $[0, T]$ and $\left[T, T^{\prime}\right]$. For brevity, we write the 
normalization constant as

$$
\begin{aligned}
M_{T^{\prime}} \triangleq & \int_{0}^{T^{\prime}}\left\langle q_{u}, \boldsymbol{e}_{i}\right\rangle\left\langle v_{u, T}, \boldsymbol{e}_{j}\right\rangle d u \\
\approx & \left\{\sum _ { \ell = 1 } ^ { K } \frac { 1 } { 2 } \Delta _ { ( \ell - 1 , \ell ) } \left(\left\langle\widehat{q}_{\ell-1}, \boldsymbol{e}_{i}\right\rangle\left(\boldsymbol{e}_{i}^{\prime} \Psi_{\ell-1, T}\right)\right.\right. \\
& \left.+\left\langle\widehat{q}_{\ell}, \boldsymbol{e}_{i}\right\rangle\left(\boldsymbol{e}_{i}^{\prime} \Psi_{\ell, T}\right)\right\} \Psi_{T, T^{\prime}} \mathbf{1} \\
& +\left\{\sum _ { \ell = K + 1 } \frac { 1 } { 2 } \Delta _ { ( \ell - 1 , \ell ) } \left(\left\langle\widehat{q}_{\ell-1}, \boldsymbol{e}_{i}\right\rangle\left(\boldsymbol{e}_{i}^{\prime} \Psi_{\ell-1, T^{\prime}}\right)\right.\right. \\
& \left.\left.+\left\langle\widehat{q}_{\ell}, \boldsymbol{e}_{i}\right\rangle\left(\boldsymbol{e}_{i}^{\prime} \Psi_{\ell, T^{\prime}}\right)\right)\right\} \mathbf{1} .
\end{aligned}
$$

$$
\begin{aligned}
{\left[\widehat{A}_{\tau+1}\right]_{(i, j)}=} & {\left[\widehat{A}_{\tau}\right]_{(i, j)} } \\
& \times\left\{\sum _ { \ell = 1 } ^ { K } \frac { 1 } { 2 } \Delta _ { ( \ell - 1 , \ell ) } \left(\left\langle\widehat{q}_{\ell-1}, \boldsymbol{e}_{i}\right\rangle\left(\boldsymbol{e}_{j}^{\prime} \Psi_{\ell-1, T}\right)\right.\right. \\
& \left.\left.+\left\langle\widehat{q}_{\ell}, \boldsymbol{e}_{i}\right\rangle\left(\boldsymbol{e}_{j}^{\prime} \Psi_{\ell, T}\right)\right)\right\} \Psi_{T, T^{\prime}} \mathbf{1} / M_{T^{\prime}} \\
& +\left[\widehat{A}_{\tau}\right]_{(i, j)}\left\{\sum _ { \ell = K + 1 } ^ { \widetilde { K } } \frac { 1 } { 2 } \Delta _ { ( \ell - 1 , \ell ) } \left(\left\langle\widehat{q}_{\ell-1}, \boldsymbol{e}_{i}\right\rangle\left(\boldsymbol{e}_{j}^{\prime} \Psi_{\ell-1, T^{\prime}}\right)\right.\right. \\
& \left.\left.+\left\langle\widehat{q} \ell_{\ell}, \boldsymbol{e}_{i}\right\rangle\left(\boldsymbol{e}_{j}^{\prime} \Psi_{\ell, T^{\prime}}\right)\right)\right\} \mathbf{1} / M_{T^{\prime}}
\end{aligned}
$$

and

$\left\langle\widehat{\lambda}_{\tau+1}, \boldsymbol{e}_{i}\right\rangle=$

$$
\begin{aligned}
& \left\{\sum _ { \ell = 1 } ^ { K } \frac { 1 } { 2 } \Delta _ { ( \ell - 1 , \ell ) } \left(N_{\ell-1}\left\langle\widehat{q}_{\ell-1}, \boldsymbol{e}_{i}\right\rangle\left(\boldsymbol{e}_{i}^{\prime} A^{\prime} \Psi_{\ell-1, T}\right)\right.\right. \\
& \left.\left.\quad+N_{\ell}\left\langle\widehat{q}_{\ell}, \boldsymbol{e}_{i}\right\rangle\left(\boldsymbol{e}_{i}^{\prime} A^{\prime} \Psi_{\ell, T}\right)\right)\right\} \Psi_{T, T^{\prime}} \mathbf{1} / M_{T^{\prime}} \\
& \quad+\left\{\sum _ { \ell = K + 1 } ^ { K + \widetilde { K } } \frac { 1 } { 2 } \Delta _ { ( \ell - 1 , \ell ) } \left(N_{\ell-1}\left\langle\widehat{q}_{\ell-1}, \boldsymbol{e}_{i}\right\rangle\left(\boldsymbol{e}_{i}^{\prime} A^{\prime} \Psi_{\ell-1, T^{\prime}}\right)\right.\right. \\
& \left.\left.\quad+N_{\ell}\left\langle\widehat{q}_{\ell}, \boldsymbol{e}_{i}\right\rangle\left(\boldsymbol{e}_{i}^{\prime} A^{\prime} \Psi_{\ell, T^{\prime}}\right)\right)\right\} \mathbf{1} / M_{T^{\prime}}
\end{aligned}
$$$$
-\left\{\sum _ { \ell = 1 } ^ { K } \frac { 1 } { 2 } \Delta _ { ( \ell - 1 , \ell ) } \left(N_{\ell-1}\left\langle A \widehat{q}_{\ell-1}, \boldsymbol{e}_{i}\right\rangle\left(\boldsymbol{e}_{i}^{\prime} \Psi_{\ell-1, T}\right)\right.\right.
$$$$
\left.\left.+N_{\ell}\left\langle A \widehat{q}_{\ell}, \boldsymbol{e}_{i}\right\rangle\left(\boldsymbol{e}_{i}^{\prime} \Psi_{\ell, T}\right)\right)\right\} \Psi_{T, T^{\prime}} \mathbf{1} / M_{T^{\prime}}
$$$$
-\left\{\sum _ { \ell = K + 1 } ^ { K + \widetilde { K } } \frac { 1 } { 2 } \Delta _ { ( \ell - 1 , \ell ) } \left(N_{\ell-1}\left\langle A \widehat{q}_{\ell-1}, \boldsymbol{e}_{i}\right\rangle\left(\boldsymbol{e}_{i}^{\prime} \Psi_{\ell-1, T^{\prime}}\right)\right.\right.
$$$$
\left.\left.+N_{\ell}\left\langle A \widehat{q}_{\ell}, \boldsymbol{e}_{i}\right\rangle\left(\boldsymbol{e}_{i}^{\prime} \Psi_{\ell, T^{\prime}}\right)\right)\right\} \boldsymbol{1} / M_{T^{\prime}}
$$$$
+N_{T^{\prime}}\left\langle\widehat{q}_{T^{\prime}}, \boldsymbol{e}_{i}\right\rangle\left\langle\widehat{v}_{T^{\prime}, T^{\prime}}, \boldsymbol{e}_{i}\right\rangle / M_{T^{\prime}}
$$

$$
\begin{aligned}
{\left[\widehat{A}_{\tau+1}\right]_{(i, j)} } & =\left[\widehat{A}_{\tau}\right]_{(i, j)} \frac{\int_{0}^{T}\left\langle q_{u}, \boldsymbol{e}_{i}\right\rangle\left\langle v_{u, T}, \boldsymbol{e}_{j}\right\rangle d u}{\int_{0}^{T}\left\langle q_{u}, \boldsymbol{e}_{i}\right\rangle\left\langle v_{u, T}, \boldsymbol{e}_{i}\right\rangle d u} \\
& \approx\left[\widehat{A}_{\tau}\right]_{(i, j)} \frac{\left[\sum_{\ell=1}^{K} \frac{1}{2} \Delta_{(\ell-1, \ell)}\left(\left\langle\widehat{q}_{\ell-1}, \boldsymbol{e}_{i}\right\rangle\left\langle\widehat{v}_{\ell-1, T}, \boldsymbol{e}_{j}\right\rangle+\left\langle\widehat{q}_{\ell}, \boldsymbol{e}_{i}\right\rangle\left\langle\widehat{v}_{\ell, T}, \boldsymbol{e}_{j}\right\rangle\right)\right]}{\left[\sum_{\ell=1}^{K} \frac{1}{2} \Delta_{(\ell-1, \ell)}\left(\left\langle\widehat{q}_{\ell-1}, \boldsymbol{e}_{i}\right\rangle\left\langle\widehat{v}_{\ell-1, T}, \boldsymbol{e}_{i}\right\rangle+\left\langle\widehat{q}_{\ell}, \boldsymbol{e}_{i}\right\rangle\left\langle\widehat{v}_{\ell, T}, \boldsymbol{e}_{i}\right\rangle\right)\right]} \\
& =\left[\widehat{A}_{\tau}\right]_{(i, j)} \frac{\left.\left[\sum_{\ell=1}^{K} \frac{1}{2} \Delta_{(\ell-1, \ell)}\left(\left\langle\widehat{q}_{\ell-1}, \boldsymbol{e}_{i}\right\rangle\left(\boldsymbol{e}_{j}^{\prime} \Psi_{\ell-1, T}\right)+\left\langle\widehat{q}_{\ell}, \boldsymbol{e}_{i}\right\rangle\left(\boldsymbol{e}_{j}^{\prime} \Psi_{\ell, T}\right)\right)\right\} \mathbf{1}\right]}{\left[\sum_{\ell=1}^{K} \frac{1}{2} \Delta_{(\ell-1, \ell)}\left(\left\langle\widehat{q}_{\ell-1}, \boldsymbol{e}_{i}\right\rangle\left(\boldsymbol{e}_{i}^{\prime} \Psi_{\ell-1, T}\right)+\left\langle\widehat{q}_{\ell}, \boldsymbol{e}_{i}\right\rangle\left(\boldsymbol{e}_{i}^{\prime} \Psi_{\ell, T}\right)\right)\right] \mathbf{1}} .
\end{aligned}
$$

$$
\begin{aligned}
& \left\langle\widehat{\lambda}_{\tau+1}, \boldsymbol{e}_{i}\right\rangle \\
& =\frac{\left[\sum_{\ell=1}^{K} \frac{1}{2} \Delta_{(\ell-1, \ell)}\left(N_{\ell-1}\left\langle\widehat{q}_{\ell-1}, \boldsymbol{e}_{i}\right\rangle\left(\boldsymbol{e}_{i}^{\prime} A^{\prime} \Psi_{\ell-1, T}\right)+N_{\ell}\left\langle\widehat{q} \ell_{\ell}, \boldsymbol{e}_{i}\right\rangle\left(\boldsymbol{e}_{i}^{\prime} A^{\prime} \Psi_{\ell, T}\right)\right)\right] \mathbf{1}}{\left[\sum_{\ell=1}^{K} \frac{1}{2} \Delta_{(\ell-1, \ell)}\left(\left\langle\widehat{q}_{\ell-1}, \boldsymbol{e}_{i}\right\rangle\left(\boldsymbol{e}_{i}^{\prime} \Psi_{\ell-1, T}\right)+\left\langle\widehat{q}_{\ell}, \boldsymbol{e}_{i}\right\rangle\left(\boldsymbol{e}_{i}^{\prime} \Psi_{\ell, T}\right)\right)\right] \mathbf{1}} \\
& +\frac{\left[\sum_{\ell=1}^{K} \frac{1}{2} \Delta_{(\ell-1, \ell)}\left(N_{\ell-1}\left\langle A \widehat{q}_{\ell-1}, \boldsymbol{e}_{i}\right\rangle\left(\boldsymbol{e}_{i}^{\prime} \Psi_{\ell-1, T}\right)+N_{\ell}\left\langle A \widehat{q}_{\ell}, \boldsymbol{e}_{i}\right\rangle\left(\boldsymbol{e}_{i}^{\prime} \Psi_{\ell, T}\right)\right)\right] \mathbf{1}}{\left[\sum_{\ell=1}^{K} \frac{1}{2} \Delta_{(\ell-1, \ell)}\left(\left\langle\widehat{q}_{\ell-1}, \boldsymbol{e}_{i}\right\rangle \times\left(\boldsymbol{e}_{i}^{\prime} \Psi_{\ell-1, T}\right)+\left\langle\widehat{q}_{\ell}, \boldsymbol{e}_{i}\right\rangle\left(\boldsymbol{e}_{i}^{\prime} \Psi_{\ell, T}\right)\right)\right] \mathbf{1}} \\
& +\frac{N_{T}\left\langle\widehat{q}_{T}, \boldsymbol{e}_{i}\right\rangle\left\langle\widehat{v}_{T, T}, \boldsymbol{e}_{i}\right\rangle}{\left[\sum_{\ell=1}^{K} \frac{1}{2} \Delta_{(\ell-1, \ell)}\left(\left\langle\widehat{q}_{\ell-1}, \boldsymbol{e}_{i}\right\rangle\left(\boldsymbol{e}_{i}^{\prime} \Psi_{\ell-1, T}\right)+\left\langle\widehat{q}_{\ell}, \boldsymbol{e}_{i}\right\rangle\left(\boldsymbol{e}_{i}^{\prime} \Psi_{\ell, T}\right)\right)\right] \mathbf{1}} .
\end{aligned}
$$


Note that the sums in these two formulas are approximating integrals and need not be completely recalculated. Write, for example,

$$
\begin{aligned}
B \triangleq\left\{\sum _ { \ell = 1 } ^ { K } \frac { 1 } { 2 } \Delta _ { ( \ell - 1 , \ell ) } \left(\left\langle\widehat{q}_{\ell-1}, \boldsymbol{e}_{i}\right\rangle\right.\right. & \left(\boldsymbol{e}_{j}^{\prime} \Psi_{\ell-1, T}\right) \\
& \left.\left.+\left\langle\widehat{q}_{\ell}, \boldsymbol{e}_{i}\right\rangle\left(\boldsymbol{e}_{j}^{\prime} \Psi_{\ell, T}\right)\right)\right\} .
\end{aligned}
$$

This $n \times n$ matrix appears in (5.89) and can be stored in memory. Upon the arrival of new information on $\left[T, T^{\prime}\right]$, the matrix $B$ can be recalled from memory and multiplied on the right-hand side by $\Psi_{T, T^{\prime}} \mathbf{1}$. Similarly, the corresponding matrix in (5.90) can be stored in memory, avoiding a complete pass through the data as in previous algorithms cited in the bibliography.

\section{APPENDIX I}

\section{PROOF OF LEMMA 1}

Proof: To establish Lemma 1, we first show that $M$ is $(P, \mathcal{F})$ martingale. Since, under $P^{\dagger}$, the process $\Lambda M$ has dynamics

$$
\Lambda_{t} M_{t}=M_{0}+\int_{0}^{t} \Lambda_{u} d M_{u}+\int_{0}^{t} M_{u} d \Lambda_{u}
$$

$$
E\left[M_{t} \mid \mathcal{F}_{s}\right]=\frac{E^{\dagger}\left[\Lambda_{t} M_{t} \mid \mathcal{F}_{s}\right]}{E^{\dagger}\left[\Lambda_{t} \mid \mathcal{F}_{s}\right]}=\frac{\Lambda_{s} M_{s}}{\Lambda_{s}}=M_{s} .
$$

Therefore,

$$
E\left[M_{t}-M_{s} \mid \mathcal{F}_{s}\right]=\mathbf{0} \in \mathbb{R}^{n}
$$

So,

$$
E\left[X_{t}-X_{s}-\int_{s}^{t} A X_{u} d u \mid \mathcal{F}_{s}\right]=\mathbf{0} \in \mathbb{R}^{n}
$$

Then,

$$
\begin{aligned}
Z_{t} & \triangleq E\left[X_{t} \mid \mathcal{F}_{s}\right]=X_{s}+\int_{s}^{t} A E\left[X_{u} \mid \mathcal{F}_{u}\right] d u \\
& =X_{s}+\int_{s}^{t} A Z_{u} d u .
\end{aligned}
$$

Therefore,

$$
Z_{t}=\exp (A(t-s)) X_{s}=E\left[X_{t} \mid \mathcal{F}_{s}\right]=E\left[X_{t} \mid X_{s}\right] .
$$

Equation (A6) shows that, under the measure $P^{\dagger}$, the process $X$ satisfies the Markov property and that its rate matrix is again $A$. To complete the proof, we note that

$$
E\left[X_{0} \mid\{\Omega, \emptyset\}\right]=\frac{E^{\dagger}\left[\Lambda_{0}^{-1} X_{0} \mid\{\Omega, \emptyset\}\right]}{E^{\dagger}\left[\Lambda_{0}^{-1} \mid\{\Omega, \emptyset\}\right]}=E\left[X_{0}\right]=p_{0} .
$$

\section{APPENDIX II}

DERIVATION OF THE STOCHASTIC INTEGRAL EQUATION (3.12)

Proof: We wish to estimate $X$ given the observations $\mathcal{Y}$ of 372 $N$. By Bayes' rule,

$$
E\left[X_{t} \mid \mathcal{Y}_{0, t}\right]=\frac{E^{\dagger}\left[\Lambda_{0, t} X_{t} \mid \mathcal{Y}_{0, t}\right]}{E^{\dagger}\left[\Lambda_{0, t} \mid \mathcal{Y}_{0, t}\right]}
$$

Note that $\left\langle X_{t}, \mathbf{1}\right\rangle=1$. So,

$$
\begin{aligned}
\left\langle E^{\dagger}\left[\Lambda_{0, t} X_{t} \mid \mathcal{Y}_{0, t}\right], \mathbf{1}\right\rangle & =E^{\dagger}\left[\Lambda_{0, t}\left\langle X_{t}, \mathbf{1}\right\rangle \mid \mathcal{Y}_{0, t}\right] \\
& =E^{\dagger}\left[\Lambda_{0, t} \mid \mathcal{Y}_{0, t}\right] .
\end{aligned}
$$

That is, if we write

$$
q_{t}=E^{\dagger}\left[\Lambda_{0, t} X_{t} \mid \mathcal{Y}_{0, t}\right]
$$

then

$$
P\left(X_{t}=\boldsymbol{e}_{i}\right) \triangleq E\left[X_{t}=\boldsymbol{e}_{i} \mid \mathcal{Y}_{0, t}\right]=\frac{1}{\left\langle q_{t}, \mathbf{1}\right\rangle}\left\langle q_{t}, \boldsymbol{e}_{i}\right\rangle .
$$

To compute the expectation at (B3), we first apply the product 377 rule to determine the decomposition for the process $\Lambda X$

$$
\begin{aligned}
\Lambda_{0, t} X_{t}= & X_{0}+\int_{0}^{t} \Lambda_{0, u} A X_{u} d u+\int_{0}^{t} \Lambda_{u-} d M_{u} \\
& +\int_{0}^{t} X_{u-}\left(\left\langle X_{u-}, \lambda\right\rangle-1\right) \Lambda_{0, u-}\left(d N_{u}-d u\right) \\
= & X_{0}+\int_{0}^{t} \Lambda_{0, u} A X_{u} d u+\int_{0}^{t} \Lambda_{u-} d M_{u} \\
& +\sum_{i=1}^{n} \int_{0}^{t}\left\langle X_{u-}, \boldsymbol{e}_{i}\right\rangle\left(\left\langle\lambda, \boldsymbol{e}_{i}\right\rangle-1\right) \Lambda_{0, u-}\left(d N_{u}-d u\right) \boldsymbol{e}_{i} .
\end{aligned}
$$

By conditioning both sides of (B5) on $\mathcal{Y}_{0, t}$ under the refer- 379 ence probability $P^{\dagger}$, it then follows that the process $q$ has the 380 dynamics

$$
\begin{aligned}
q_{t}= & q_{0}+\int_{0}^{t} A q_{u} d u \\
& +\int_{0}^{t} \operatorname{diag}\left\{\left\langle\lambda, \boldsymbol{e}_{\ell}\right\rangle-1\right\} q_{u-}\left(d N_{u}-d u\right) .
\end{aligned}
$$

\section{APPENDIX III}

\section{Proof OF THEOREM 3}

To compute the dynamics of the process $\sigma\left(G^{i} X\right)$, we must 384 evaluate the expectation $E^{\dagger}\left[\Lambda_{t} G_{t}^{i} X_{t} \mid \mathcal{Y}_{0, t}\right]$. Using the product rule, we compute the decomposition of the process $G X \Lambda$

$$
\begin{aligned}
& \Lambda_{t} G_{t}^{i} X_{t} \\
& =\int_{0}^{t} \Lambda_{u-} X_{u}\left\langle X_{u}, \boldsymbol{e}_{i}\right\rangle d N_{u}+\int_{0}^{t} \Lambda_{u} G_{s}^{i} A X_{u} d u \\
& \quad+\int_{0}^{t} \Lambda_{u} G_{u}^{i} d M_{u}+\int_{0}^{t} G_{u}^{i} X_{u} \Lambda_{u-}\left(\left\langle X_{u}, \lambda\right\rangle-1\right)\left(d N_{u}-d u\right) \\
& \quad+\int_{0}^{t} X_{u}\left\langle X_{u}, \boldsymbol{e}_{i}\right\rangle \Lambda_{u-}\left(\left\langle X_{u}, \Lambda\right\rangle-1\right)\left(d N_{u}-d u\right) .
\end{aligned}
$$




$$
\begin{aligned}
E^{\dagger}\left[\Lambda_{t}\right. & \left.G_{t}^{i} X_{t} \mid \mathcal{Y}_{0, t}\right] \\
= & \int_{0}^{t} E^{\dagger}\left[\Lambda_{u-} X_{u}\left\langle X_{u}, \boldsymbol{e}_{i}\right\rangle \mid \mathcal{Y}_{0, s}\right] d N_{u} \\
& +\int_{0}^{t} E^{\dagger}\left[\Lambda_{u} G_{u}^{i} A X_{u} \mid \mathcal{Y}_{0, s}\right] d u+\int_{0}^{t} E^{\dagger}\left[\Lambda_{u} G_{u}^{i} \mid \mathcal{Y}_{0, s}\right] d M_{u} \\
& +\int_{0}^{t} E^{\dagger}\left[G_{u}^{i} X_{u} \Lambda_{u-}\left(\left\langle X_{u}, \lambda\right\rangle-1\right) \mid \mathcal{Y}_{0, s}\right]\left(d N_{u}-d u\right) \\
& +\int_{0}^{t} E^{\dagger}\left[X_{u}\left\langle X_{u}, \boldsymbol{e}_{i}\right\rangle \Lambda_{u-}\left(\left\langle X_{u}, \Lambda\right\rangle-1\right) \mid \mathcal{Y}_{0, s}\right]\left(d N_{u}-d u\right) \\
= & \int_{0}^{t} A \sigma\left(G_{u}^{i} X_{u}\right) d u \\
& +\int_{0}^{t} \operatorname{diag}\left\{\left\langle\lambda, \boldsymbol{e}_{\ell}\right\rangle-1\right\} \sigma\left(G_{u}^{i} X_{u}\right)\left(d N_{u}-d u\right) \\
& +\int_{0}^{t}\left\langle q_{u}, \boldsymbol{e}_{i}\right\rangle\left\langle\lambda, \boldsymbol{e}_{i}\right\rangle d N_{u} \boldsymbol{e}_{i} .
\end{aligned}
$$

\section{ACKNOWLEDGMENT}

The authors would like to thank the anonymous reviewers, whose suggestions have greatly improved the manuscript.

W. P. Malcolm would also like to acknowledge: Prof. D. J. Daley, for his helpful technical discussions concerning the doubly stochastic Poisson processes.

\section{REFERENCES}

[1] J. M. C. Clark, "The design of robust approximations to the stochastic differential equations for nonlinear filtering," in Communications Systems and Random Process Theory, J. K. Skwirzynski, Ed. Alphen aan den Rijn, The Netherlands: Sijthoff and Noorhoff, 1978, pp. 721-734.

[2] R. J. Elliott, Stochastic Calculus and its Applications. New York: Springer-Verlag, 1982.

[3] L. Aggoun and R. J. Elliott, Measure Theory and Filtering. Cambridge, U.K.: Cambridge Univ. Press, 2004.

[4] R. J. Elliott, "New finite dimensional filters and smoothers for noisily observed markov chains," IEEE Trans. Inf. Theory, vol. 39, no. 1, pp. 265271, Jan. 1993.

[5] P. Bremaud, Point Processes and Queues, Martingale Dynamics. New York: Springer-Verlag, 1981.

[6] F. Campillo and F. L. Gland, "Maximum likelihood estimation for partially observed diffusions: Direct maximization versus the em algorithm," Stochastic Processes Appl., vol. 33, no. 2, pp. 245-274, 1989.

[7] A. Dembo and O. Zeitouni, "Parameter estimation of partially observed continuous time stochastic processes via the em algorithm," Stochastic Processes Appl., vol. 23, pp. 91-113, 1986.

[8] A. D. Demster, N. M. Laird, and D. B. Rubin, "Maximum likelihood from incomplete data via the em algorithm," J. Royal Statist. Soc., vol. B-39, pp. 1-37, 1997.

[9] R. J. Elliott, "Exact adaptive filters for Markov chains observed in Gaussian noise," Automatica, vol. 30, no. 9, pp. 1399-1408, Sep. 1994.

[10] M. R. James, V. Krishnamurthy, and F. L. Gland, "Time discretization for continuous time filters and smoothers for hmm parameter estimation," IEEE Trans. Inf. Theory, vol. 42, no. 2, pp. 593-605, Mar. 1998.

[11] F. Konecny, "Parameter estimation for point processes with partial observations: Afiltering approach," Syst. Control Lett., vol. xx, no. 4, pp. 281286,1984
[12] G. Lindgren and U. Holst, "Recursive estimation of parameters in Markovmodulated Poisson processes," IEEE Trans. Commun., vol. 43, no. 11, pp. 2812-2820, Nov. 1995.

[13] W. P. Malcolm, "Robust filtering and estimation with Poisson observations" Ph.D. dissertation, The Australian Nat. Univ., Canberra, Australia, 1999.

[14] W. P. Malcolm and R. J. Elliott, "A general smoothing equation for Poisson observations," presented at the IEEE Conf. Decision Control, Phoenix, AZ, Dec. 1999.

[15] R. J. Elliott and W. P. Malcolm, "Robust smoother dynamics for Poisson processes driven by an itô diffusion," presented at the IEEE Conf. Decision Control, Orlando, FL, Dec. 2001.

16] W. P. Malcolm, R. J. Elliott, and J. van der Hoek, "On the numerical 440 stability of time-discretized state estimation via clark transformations," 441 presented at the IEEE Conf. Decision Control, Mauii, HI, Dec. 2003.

[17] J. McLachlan and T. Krishnan, The EM Algorithm and Extensions. New 443 York: Wiley, 1997.

[18] E. Pardoux, "Equations du filtrage nonlineare de la predictions et du lissage," Stochastics, vol. xx, no. 6, pp. 193-231, 1982.

[19] E. Pardoux and P. Protter, "A two-sided stochastic integral and its calculus," Probab. Theory Relat. Fields, vol. 76, pp. 15-49, 1987.

[20] H. Royden, Real Analysis, 3rd ed. New York: MacMillan, 1988.

[21] T. Ryden, "Parameter estimation for markov modulated Poisson processes," Commun. Statist. Stochastic Models, vol. 10, no. 4, pp. 795-829, 1994.

[22] T. Ryden, "An em algorithm for estimation in Markov-modulated poisson processes," Comput. Statist. Data Anal., vol. 21, pp. 431-447, 1996.

[23] A. Segall, M. H. A. Davis, and T. Kailath, "Nonlinear filtering with counting process observations," IEEE Trans. Inf. Theory, vol. 21, no. 2, pp. 143149, Mar. 1975.

[24] R. H. Shumway and D. S. Stoffer, "An approach to time series smoothing and forecasting using the em algorithm," J. Time Ser. Anal., vol. 3, no. 4, pp. 682-687, 1989 .

[25] D. L. Snyder, "Filtering and detection for doubly stochastic poisson processes," IEEE Trans. Inf. Theory, vol. 18, no. 1, pp. 91-102, Jan. 1972.

[26] D. L. Snyder, "Smoothing for doubly stochastic poisson processes," IEEE Trans. Inf. Theory, vol. 18, no. 5, pp. 558-562, Sep. 1972.

[27] E. Wong and B. Hajek, Stochastic Processes in Engineering Systems. New York: Springer-Verlag, 1985. of transitions of finite-state continuous time Markov processes," IEEE Trans. Inf. Theory, vol. 34, no. 4, pp. 890-893, Jul. 1988.

29] H. H. Ziskind, "Maximum likelihood localization of narrow band sources via the em algorithm," presented at the IEEE Int. Conf. Acoust. Speech Signal Process., Minneapolis, MN, Apr. 1993.

[30] L. Deng and J. W. Mark, "Parameter estimation for Markov modulated Poisson processes via the em algorithm with time discretization," Telecommun. Syst., vol. 1, pp. 321-328, 1993.

Robert J. Elliott received the Bachelor's and Master's degrees from Oxford 476 University, Oxford, U.K., and the Ph.D. and D.Sc. degrees from Cambridge University, Cambridge, U.K.

He has held positions at Newcastle, Yale, Oxford, Warwick, Hull, and Alberta, and visiting positions in Toronto, Northwestern, Kentucky, Brown, Paris, Denmark, Hong Kong, and Australia. He is currently the AF Collins Professor of Finance at the University of Alberta, Edmonton, Canada, and also jointly holds a position in the Mathematical Sciences. He is a frequent visitor to Adelaide. He is the author or coauthor of more than 250 papers published in various international journals and six books, including Mathematics of Financial Markets (Springer). His current research interests include stochastic processes in engineering and finance.

W. P. Malcolm received the Bachelor's degree in applied physics from the Royal Melbourne Institute of Technology, Malbourne, Australia, the Master's degree in applied mathematics from the Flinders University, Adelaide, South Australia, and the Ph.D. degree in electrical engineering from the Australian National University, Canberra, Australia.

He is currently a Senior Researcher in the Statistical Machine Learning Program of the National ICT Australia (NICTA), Canberra, Australia. His current research interests include filtering, estimation, stochastic control, and measures of risk in decision making with uncertainty. In particular, filtering and stochastic control with point process observations and marked point process observations.
431

434

435

436

437

443

444

445

446 449

450

451

453

455
456

457

458

459

460

461

463

464

465

466
467

468 469

471

473

474

475

477

(4)

479 480 
Q1. Author: Please provide the IEEE membership details, if any, for both the authors.

Q2. Author: Fraction given in solidus form in Eq. (5.90) have been set as display fraction. Please check if equation is ok as typeset. 502

Q3. Author: Please provide the subjects and the years in which R. J. Elliott received the Bachelor's, Master's, Ph.D. and D.Sc. 503 degrees. Also, please provide the names of the institutes in Newcastle, Yale, Oxford, Warwick, Hull, Alberta, Toronto, 504 Northwestern, Kentucky, Brown, Paris, Denmark, Hong Kong, and Australia, where he held various positions.

Q4. Author: Please provide the years in which W. P. Malcolm received the Bachelors, Masters, and Ph.D. degrees. 